\title{
Nanotecnología aplicada a la salud pública
}

\author{
Pascual Gerardo García Zamora* \\ Dellanira Ruiz de Chávez Ramírez**
}

En el presente artículo se exponen las aplicaciones de las nanotecnologías al sector médico. Al mismo tiempo se alerta sobre los potenciales riesgos en un contexto donde el objetivo inmediato de las empresas es la obtención de ganancia, lo que puede tergiversar el propósito de mejorar la salud humana.

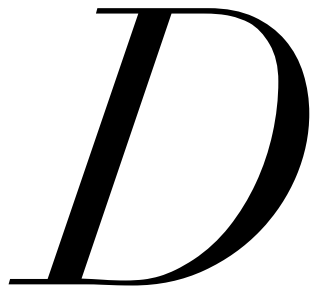

ESDE EL MOMENTO en que los seres humanos utilizaron el primer instrumento para alcanzar un fruto y satisfacer su apetito, se inicia el proceso de desarrollo de tecnologías para lograr una mayor y mejor adaptación al medio ambiente. Al inicio, el objetivo era conocer y entender los fenómenos que se presentaban

* Coordinador de la maestría en Ciencias de la Salud de la de la Unidad Académica de Medicina Humana y Ciencias de la Salud de la Universidad Autónoma de Zacatecas, México. Correo electrónico: ggaza2ooo@yahoo.com.mx.

** Docente-investigadora de la Unidad Académica de Medicina Humana y Ciencias de la Salud de la Universidad Autónoma de Zacatecas, México. en la naturaleza; después de muchos siglos se logra acumular conocimiento de los ciclos agrícolas, y de esa manera se desarrolla ya cierto control sobre la producción de gramíneas y hortalizas para consumo humano, y no es sino hasta la Revolución industrial cuando el desarrollo del conocimiento y la tecnología rebasan la capacidad de recuperación de la naturaleza y aparece el humano con un mayor control sobre la misma.

La salud y enfermedad evolucionan desde concepciones instintivas a las mágico-teológicas concebidas durante la Edad media, gracias al desarrollo de las tecnologías y a un conocimiento más preciso de la anatomía y fisiología de los seres vivos donde el hombre aspira a intervenir en procesos mórbidos del ser humano ante la negativa de la visión teológica. En el siglo 
XIX se conjuga y sistematiza toda la información acumulada hasta este momento, dando paso a la conformación de la visión microbiológica de la enfermedad. El siglo xx impulsa el desarrollo de la industria farmacológica para la generación de productos que ayuden al combate de los agentes etiológicos, causantes de enfermedades infecto-contagiosas, además de productos químicos para el saneamiento básico de zonas insalubres y pantanosas.

La práctica de la salud pública se enfoca principalmente en el monitoreo y control de enfermedades infecciosas con una medicina clínica basada en la concepción sistémica de la naturaleza, influencia importada a partir de la física del Renacimiento donde el cuerpo humano se conforma por aparatos y sistemas, siendo la célula la parte más pequeña de todo ser vivo. Más tarde, Watson y Crick descubren que el ácido desoxirribonucleico (DNA, por sus siglas en inglés) es una molécula de doble hélice donde la estructura medular de cada hebra está conformada con la alternancia de azúcares (ribosa) y fosfato, y que entre estas dos hebras existe un apareamiento de bases nitrogenadas unidas mediante puentes de hidrógeno. La secuencia de esas bases nitrogenadas provee la información genética que cada individuo tiene dentro de su propio genoma, y es precisamente uno de los blancos moleculares donde inciden las aplicaciones nanotecnológicas. ${ }^{1}$

Ya Demócrito y Leucipo en el siglo v a. C. señalaron que la materia está constituida por átomos de diferente magnitud, peso, forma y postura; sin embargo, la posibilidad de comprobarlo sucede hasta el siglo xx." "Manipular y controlar cosas en la escala de lo pequeño", decía Richard Feyman ${ }^{3}$ al explicar que ninguna ley física evitaba que se pudieran hacer cosas como miniaturizar las computadoras y escribir la información contenida en todos los libros en un cubo del tamaño de una partícula de polvo y construir máquinas con átomos y moléculas. Esto abre todo un universo de posibilidades para la intervención de la especie humana en todos los procesos naturales, sociales y su impacto en la calidad de vida.

\section{Aplicaciones de la nanotecnología}

Los avances científico-tecnológicos se perciben en forma más evidente en las tecnologías de la información y la comunicación, pero poco a poco vemos cómo se va innovando en la industria aeroespacial y automotriz. Los procesos de curación no se quedan al margen.
La industria farmacéutica encuentra nichos de oportunidad en la generación de nuevos medicamentos de larga biodisponibilidad y otros de acción localizada, gracias a intervenciones previas de nanopartículas inmunofluorescentes. Además, se comienza a trabajar con otras que aceleran los procesos de consolidación de fracturas y cicatrización de heridas, como si se tratara de un proceso natural, pero más acelerado y con mayor eficacia. Para el diagnóstico de enfermedades se desarrollan tecnologías no invasivas; además, con el uso del nanorobot, con menor agresividad que las técnicas endoscópicas, se pueden localizar células cancerígenas a poco tiempo de iniciar su desarrollo así como apoyar en la vigilancia de la homeostasis del cuerpo humano.

En la salud de las colectividades se utilizan nanopartículas de metales bajo una estrategia general que consiste en colocar nanotransportadores o nanomateriales que ayudan a mejorar la absorción y biodisponibilidad de sustancias nutritivas agregadas como vitaminas y minerales, denominados materiales inteligentes. ${ }^{4}$ Otro uso de la nanotecnología en la industria alimentaria guarda relación con los materiales de contacto de los alimentos. ${ }^{.}$En la actualidad algunos nanocompuestos son usados como material de embalaje o recubrimiento para controlar la difusión de gases y prolongar el tiempo de conservación de diversos productos. Con mayor frecuencia se utilizan más compuestos basados en la nanotecnología para elaborar materiales de contacto con los alimentos, los cuales además están dotados de propiedades antimicrobianas. Las actuales investigaciones sobre ese tipo de superficie tienen por objeto conseguir sensores capaces de detectar la contaminación bacteriana y reaccionar contra ella. ${ }^{6}$

La nanotecnología es un área emergente que nos está conduciendo a una nueva era científico-tecnológica. Entre las aplicaciones más prometedoras y dinámicas están aquellas del sector salud, donde destacan las investigaciones en enfermedades como el cáncer, VIH SIDA, diabetes, osteoartritis, enfermedades degenerativas y desórdenes cardiovasculares y nerviosos.? Hoy existen nuevos y numerosos sistemas nanoestructurados con múltiples aplicaciones potenciales en el área biomédica en los cuales, además de la caracterización fisicoquímica (tamaño de partículas, potencial zeta, solubilidad estabilidad, etcétera), es necesario estudiar su interacción con los sistemas biológicos. Asimismo, se requiere conocer su capacidad de ingresar en las células el mecanismo de acción, condiciones 
de metabolización e integración con las nanobiomoléculas y, desde luego, conocer también el daño que pueden provocar a nivel celular o del organismo.

Un conocimiento detallado de la interacción de los sistemas nanoestructurados con los sistemas biológicos conducirá al desarrollo de sistemas biocompatibles. Este conocimiento permitirá desarrollar nuevas terapias para el tratamiento de enfermedades como parto prematuro, cáncer, enfermedades neurodegenerativas, entre otras; cuando éstas se encuentran en estados poco avanzados o al inicio de su desarrollo, se hace uso de dispositivos capaces de interactuar a nivel molecular con respuestas a nivel celular.

Una razón para estudiar la biología a nanoescala es poder estudiar propiedades que no se ven en escalas micro o macro. Por ejemplo, medir propiedades físicas de proteínas individuales nos puede dar información sobre su estructura y función. Esta información sin duda ayudará a entender cómo los diferentes componentes de un sistema biológico trabajan juntos. También ayudarán al uso de bionanoestructuras y nanodispositivos para sistemas y procedimientos mejorados y novedosos de prevención, diagnóstico, tratamiento y regeneración, los cuales constituyen el corazón de la denominada "nanomedicina", refiriéndose precisamente al cruce de la nanotecnología y la medicina que alude esencialmente al encuentro de éstas en el amplio ámbito de la salud, la última con mayor tendencia hacia la denominada medicina personalizada.

\section{Nanomedicina}

La nanomedicina agrupa tres áreas principales: a) el nanodiagnóstico, en el cual se han desarrollado sistemas de análisis e imágenes que pueden detectar anormalidades en las estructuras celulares en estadios tempranos; b) la nanoterapia, la cual contribuye a crear terapias eficaces y específicas dirigidas especialmente a tratar células alteradas sin dañar las sanas así como disminuir los efectos secundarios; y c) la nanomedicina regenerativa, donde el objetivo principal es el reemplazo de órganos o tejidos dañados con la ayuda de herramientas nanotecnológicas. ${ }^{8}$

\section{Nanodiagnóstico}

El principal objetivo del nanodiagnóstico es la detección temprana de una enfermedad a través de una o muy pocas moléculas. Las herramientas de nanodiag- nóstico en el laboratorio de análisis clínico están constituidas por nanocristales semiconductores que se emplean como una etiqueta fluorescente; entre ellas se encuentran los puntos cuánticos utilizados para pruebas inmunohistoquímicas para la detección de neurotransmisores e imágenes celulares; soportes o "cantilevers" aplicados para la detección y cuantificación de proteínas y de DNA, y el oro en partículas nanométricas, el cual se utiliza para la detección de agentes infecciosos por hibridación de DNA. 9

Las nuevas técnicas de diagnóstico por imagen a través de trazadores y nuevos agentes de contraste (formados por nanopartículas sintéticas y biológicas) ayudan a visualizar las causas que producen una enfermedad pero a escala molecular; para ello se han desarrollado nuevas técnicas con una mayor resolución y sensibilidad para diagnósticos en estadios tempranos en casos como el cáncer, enfermedades cardiovasculares y síndromes neurológicos. ${ }^{10}$ Otras aplicaciones se refieren a la mejora del propio instrumental médico, del equipo de resolución molecular ultra avanzado para la generación de nanoimágenes, útiles para diagnósticos más precisos, procedimientos novedosos de diagnóstico in vivo e in vitro, uso de biosensores para diagnóstico, tratamiento y posterapia, así como a la mejora de diversos dispositivos portátiles de diagnóstico o monitoreo.

Nanoterapia o liberación de fármacos

La liberación de fármacos cuenta con un sistema transportador directo al sitio específico (órganos, tejidos o células dañadas) en las cantidades apropiadas a través de dosis requeridas. Las características con las que deben de cumplir es una baja toxicidad, óptimas condiciones para el transporte y liberación con un mayor tiempo de vida media. Los dispositivos que se utilizan son en escalas nanométricas para facilitar el ingreso a la membrana celular. Dentro de éstos se encuentran las micelas, liposomas, dendrímeros, nanopartículas, nanotubos y los conjugados poliméricos." Los nuevos tratamientos para tumores cerebrales consisten en inyectar nanopartículas magnéticas en la zona del tumor que producen un calentamiento local con la destrucción de las células cancerosas. ${ }^{2}$

Nanomedicina regenerativa

La nanomedicina regenerativa está enfocada a la sustitución de órganos o tejidos dañados mediante la aplicación de terapia génica, celular e ingeniería tisular, las cuales pueden permitir una mejor integración de los 

y adultas, así como la producción de moléculas bioactivas. Las principales aportaciones consideran nuevos materiales y sistemas de soporte tales como nanotubos de carbono, nanopartículas de hidroxiapatita o zirconia, nanofibras de polímeros biodegradables, etcétera. En la Universidad de Harvard están trabajando con fibras de ro nanómetros (nanofibers) para los casos de enfermedad cardiovascular de infartos extensos, las cuales favorecen el desarrollo del músculo cardiaco en las zonas de infarto. ${ }^{33}$ También existe desde la encapsulación de drogas y el desarrollo de procesos de entrega de las mismas para la regeneración de tejidos como hueso, cartílago, piel y nervios hasta tratamientos más sofisticados que junto con los anteriores se les denomina medicina integrativa; esta última es posible gracias al desarrollo de biomateriales e implantes biológicoinherentes, bioactivos o inteligentes y adaptativos incluyendo los implantes retinales, auditivos, de espina dorsal y de cerebro. ${ }^{14}$

Los avances que muestra la nanomedicina podrán ayudar a realizar mejores diagnósticos y tratamientos de mayor eficacia, contribuyendo a una mejor calidad de vida para la humanidad. En pleno siglo XXI, el desarrollo de las nanotecnologías abre un universo de posibilidades en la aplicación de la vida humana, con un mayor enfoque a intervenir en las enfermedades (no importa el estadio de desarrollo) con las mismas pautas de crecimiento que hasta ahora lo han hecho los medicamentos, orientados fundamentalmente al aspecto curativo de múltiples enfermedades crónicodegenerativas, y cada vez menos en lo preventivo o en resolver enfermedades infecto-contagiosas, además de incrementar desmesuradamente los productos denominados lifestyle, que son medicamentos para bajar de peso, acabar con las arrugas, dejar de fumar, combatir la impotencia o la alopecia. Todas esas píldoras que no curan ni tratan patologías, amenazan seriamente a la salud y aumentan las ventas a un ritmo dos veces superior al resto del sector farmacéutico. ${ }^{25} \mathrm{La}$ industria farmacéutica está más interesada en promover medicamentos novedosos y caros, que en muchas ocasiones resultan menos eficaces que otros ya conocidos. El objetivo es mantener, administrar, jugar con la enfermedad, pues su tratamiento produce beneficios económicos; asimismo impulsa una creciente "medicalización de experiencias transitorias y biológicas" cuyo objetivo es abrir nuevos mercados. ${ }^{16}$
Desde la perspectiva social de la salud pública, dos hitos importantes en la evolución del mundo fueron planteados para resolver problemas de enfermedades infecciosas: las propuestas de Johann Peter Frank y posteriormente Rudolf Virchow (s. XIX). En ambos casos se proponían mejoras económicas, libertad y participación social para las personas más vulnerables. Desde entonces quedó claro que hablar de salud era referirse a los elementos que determinan esta condición del ser humano, la cual se encuentra más allá de la esfera biológica de la vida misma. Al respecto, Noela Invernizzi y Guillermo Foladori ${ }^{`}$ llegan a la conclusión de que "para poder satisfacer las necesidades de los pobres, la tecnología debe usarse en un contexto socioeconómico favorable", de lo contrario seguirán las tendencias económicas dominantes aumentando las brechas de desigualdades y, por ende, la amenaza de una brecha nanotecnológica global.

Donald Maclurcan ${ }^{18}$ maneja una posición alternativa en el manejo de las nanotecnologías con equidad y en beneficio de la salud global. Vincula el desarrollo de las nanotecnologías con los Objetivos de Desarrollo del Milenio (ODM) que tienen una relación directa con la salud: ODM I, Erradicación de la extrema pobreza y el hambre; ODM 4, Reducción de la mortalidad infantil; ODM 5, Mejorar la salud materna y ODM 6 , Combate al VIH/SIDA, malaria, y otras enfermedades. Además, el suministro de aguas más higiénicas, diagnóstico rápido y preciso de enfermedades, y la creación de sistemas más eficientes de entrega de medicamentos, así como el suministro de energías limpias, reduciendo las enfermedades respiratorias causadas por el uso de combustibles no tan limpios, y el ODM 7, Garantizar la sostenibilidad del ambiente. ${ }^{19}$

El surgimiento de las nanotecnologías remite a aquellas afirmaciones que se hicieron sobre la biotecnología y sus intenciones de abordar las inequidades globales, lo que representa desafíos considerables. El primero, presentar la nanotecnología ofreciendo "soluciones" exitosas para los ODM al reducir la compleja naturaleza sociopolítica que conllevan los desafíos de salud. Sus retos demandan, más que "arreglos" tecnológicos, conciencia de los sesgos de género, geográficos, culturales, sociales, filosóficos y religiosos, que se crean alrededor de las tecnologías durante las distintas fases de la investigación y el desarrollo. Un segundo desafío para los ODM, que el aprovechamiento de las 
nanotecnologías representa, es en gran parte el proceso de transferencia de éstas desde escenarios ricos y desarrollados hacia los pobres y de escaso desarrollo.

Los debates internacionales dicen poco sobre el potencial para el desarrollo local de los pueblos al apropiarse de las nanotecnologías. De esta manera, los enfoques dominantes que conectan a la nanotecnología con los ODM perpetúan el déficit de reflexión sobre la salud internacional, la tecnología y las políticas de desarrollo.

Por otro lado, la temática desarrollada en la Agenda para el Desarrollo post 20i5, destaca la salud, hambre, nutrición, seguridad alimentaria, desigualdades, energía y agua; de ahí que las nanotecnologías también deben coadyuvar en asegurar la calidad y productividad de los alimentos, con la optimización del riego, combate de plagas para eficientar la cosecha y transformar los productos hasta el envasado y comercialización. ${ }^{20}$

Son múltiples los retos que aún se tienen en la nanomedicina. No se cuenta con una legislación amplia y sólida, las normas de reglamentación de los actuales sistemas de salud no cuentan con información sobre el uso de la nanomedicina en el diagnóstico o tratamiento; existen elementos de seguridad y efectos secundarios a largo plazo poco aclarados, además de no saber quién definirá lo que es ético. ${ }^{21}$ Por lo tanto, se requiere de evaluaciones científicas, sociales y económicas a fin de tomar decisiones sobre los riesgos y beneficios, sobre el valor final en el uso de la nanomedicina. Resulta paradójico que las mismas propiedades que convierten a las nanopartículas en materiales tan atractivos por su movilidad y habilidad para penetrar la membrana celular, también pueden ser cualidades que las conviertan en agentes sumamente peligrosos.

Desde la lógica de mejorar las condiciones de vida de las poblaciones, las nanotecnologías deben ayudar a garantizar la inocuidad, acopio, tratamiento y reciclado del agua como vital líquido para uso doméstico y agrícola. El medio ambiente es otra de las áreas de injerencia de las nanotecnologías y no menos importante para la salud de todos los seres vivos del planeta. Desde 2008, la Organización Mundial de la Salud comienza a desarrollar programas para concientizar a la población mundial de los peligros que supone el cambio climático, centrando su atención en el uso de energías, el transporte y su impacto en la salud y enfermedad de las poblaciones. El objetivo general es mejorar la salud y, a la vez, controlar los contaminantes que afectan al medio ambiente. ${ }^{22}$

Las nanotecnologías, desde la salud ambiental, deben de estar presentes en el monitoreo, reparación y purificación del medio ambiente. Desarrollo, aplicación y divulgación de uso de energías alternativas más amigables con el entorno físico y la salud de todas las especies vivas que habitamos el planeta.

\section{Conchusión}

Al hablar de nanotecnologías y salud se debe pensar en la alta relación que guardan con los determinantes sociales de la salud-enfermedad, los cuales trascienden lo biológico garantizando un uso global y no personalizado, nanotecnologías que mejoren los estilos de vida y colaboren en disminuir las brechas en la calidad de la alimentación y la disponibilidad de agua a través de un medio ambiente saludable, que además de restablecer la salud ayuden a tratar enfermedades tanto infecto-contagiosas como crónico-degenerativas. Las nanotecnologías en lo general y la nanomedicina en lo particular deben ser patrimonio de la humanidad y usarse en beneficio de la vida, por lo tanto se debe normar y vigilar su uso, para no repetir los mismos patrones de la industria farmacéutica. Por ello, las políticas públicas de todos los países deben contemplar a las nanotecnologías como una estrategia más para garantizar el desarrollo humano y la equidad.

\section{- notas $\cdot-$}

' J. D. Watson (I968), The Doublé Hélix: A Personal Account of the Discovery of the Structure of DNA, Nueva York, Atheneum.

${ }^{2}$ Federico Ortiz Quezada (I985), La enfermedad y el hombre, México, Editorial Nueva Imagen, p. 25.

${ }^{3}$ N. Takeuchi (2009), Nanociencia y nanotecnología: la construcción de un mundo mejor átomo por átomo, México, Fondo de Cultura Económica, p. 48.
${ }^{4}$ G. Foladori 2007, "Nanotecnología, salud y pobreza. ¿Cuáles son las expectativas?", Varia biológica. Filosofía, ciencia y tecnología, p. 46.

${ }^{5}$ A. G. Noormans (2010), "Impacto de la nanotecnología en la producción de alimentos", Lampsakos, 4, p. 29.

${ }^{6}$ Ibidem, p. 3i.

7 H. F. Pastrana (2007), "Nanomedicina: Estado del Arte", Revista de Ingeniería, Universidad de los Andes, 25, pp. 63-65. 
${ }^{8}$ L. M Lechuga, y A. C. Martínez (2006), "Nanobiotecnología: avances diagnósticos y terapéuticos. Especial Nanociencia y Nanotecnología”, Revista de Investigación en Gestión de la Ciencia y la Tecnología, Nanociencia y Nanotecnología II, 35, p. 3.

9 Juan M. Castagnino (2007), "Avances nanotecnológicos en Bioquímica Clínica”, Acta Bioquímica Clínica Latinoamericana, 4I:I, p. I.

${ }^{\circ}$ J. M. González, M. López y G. Ruiz (2007), Informe de vigilancia tecnológica en nanomedicina, Círculo de innovación en biotecnología, Comunidad de Madrid, 2007, http://nihroadmap. nih.gov, p. 20.

"Ibidem, p. 24.

${ }^{12}$ D. K. Chatterjee y Y. Zhang (2007), "Multi-funcional nanoparticles for cáncer therapy", Science and Technology of Advanced Materials, 8, pp. $\mathbf{1 3}_{2}$.

${ }^{13}$ H.F. Pastrana, op. cit., p. 62.

ᄂ G. C. Delgado \& L. A. Hernández (2013), "Avances e implicaciones ético-sociales de la nanomedicina: una revisión desde el caso del cáncer cerebral", Mundo Nano, Universidad Nacional Autónoma de México, 6:ı, p. 63.

${ }^{15}$ Migue Jara (2007), Traficantes de salud, Barcelona, p. ı5o.
${ }^{16}$ Ibidem, p. I8I.

${ }^{7} \mathrm{~N}$. Invernizzi y G. Foladori (2005), "Nanotechnology and the developing world: will Nanotechnology Overcome Poverty or widen disparities?", Nanotechnology Law \& Business Journal, 2:3, pp. $657-663$.

${ }^{18}$ D. C. Maclurcan, (2005), "Nanotechnology and Developing Countries, Part 2: What Realities?", AZoNano, Journal of Nanotechnology, I9 de octubre de 2005, disponible en http:// www.azonano.com/Details.asp? ArticleID $=\mathrm{I} / 429$

${ }^{19}$ Naciones Unidas (2013), Objetivos de Desarrollo del Milenio Informe 2013, Nueva York, disponible en http://www.un.org/es/ millenniumgoals/pdf/mdg-report-2or3-spanish.pdf, p. I.

${ }^{20}$ Naciones Unidas (2012), Agenda para el Desarrollo después de 2015, Nueva York. Disponible en http:/www.un.org/es/development/desa/area-of-work/post2or5.shtml, p. I.

"J.A. Álvarez, "Retos de la bioética en la medicina del siglo xxi, Revista Peru Med Exp Salud Pública, 28:4, p. 660.

22. Organización Mundial de la Salud (2014), Conferencia de OMS sobre salud y clima Ginebra Suiza, disponible en: http:/ www.who.int/globalchange/mediacentre/events/climate-healthconference/es/, p. I.

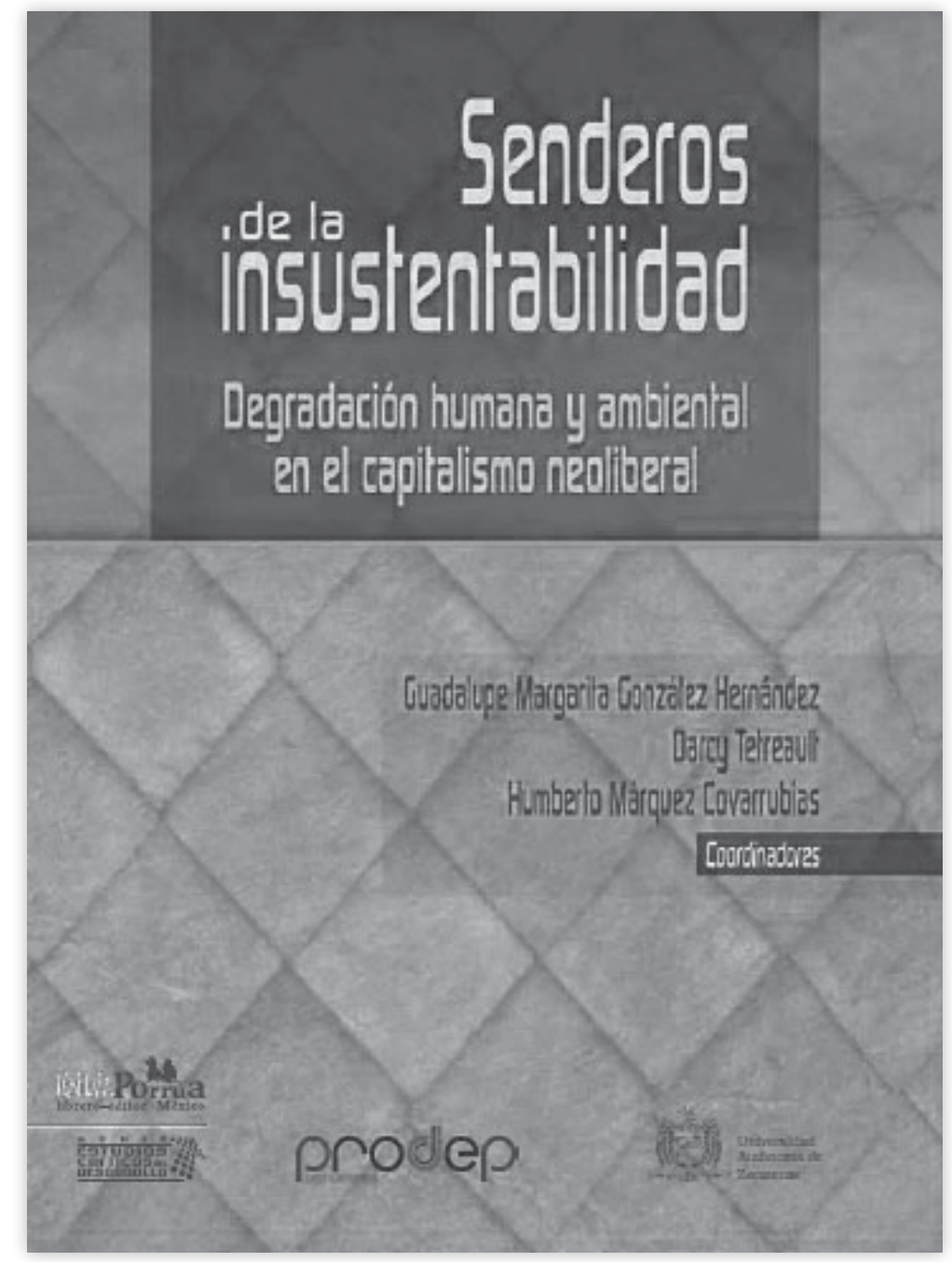

